\title{
Almost everything you wanted to know about chromosomes
}

\section{R.A. Laskey}

Eucaryotic Chromosomes. Gene Expression, Vol.2, 2nd Edn. By Benjamin Lewin. Pp.1,160. ISBN pbk 0-471-01977-1; ISBN hbk 0-471-01976-3. (Wiley: 1981.) Hbk $£ 33.70, \$ 60.65$; pbk £13.25, \$29.

THE AMOUNT of literature on cell biology is exploding at a frightening rate, so do we need this massive book? Not only is the answer yes, but I believe that this is the most valuable block of eleven hundred pages in the current cell biology literature. A vast amount of recent information has been selected, digested and evaluated to produce a unique analysis of this rapidly expanding field.

The contents provide an accurate account of research on the eukaryotic chromosome up to the end of 1979 . New discoveries in the six years between the first and second editions included the nucleosome subunits of chromatin and intervening sequences interrupting genes. These alone would justify a second edition, but a mass of detailed information on genome structure and organization has also emerged as a result of advances in recombinant DNA technology and DNA sequencing techniques. Therefore, this new edition is not just a minor revision but essentially a new book, being over twice the length of its predecessor.

The first part deals with cell structure and genetics. Surprisingly for a book entitled Eucaryotic Chromosomes, the first 70 pages provide a discussion of the cytoskeleton. However this unexpected bonus is a good introduction to the mitotic and meiotic apparatus, which are also discussed in this part together with the cell cycle and cell fusion studies. Part 2 deals with the organization of the genetic apparatus, concentrating on structure of chromatin and chromosomes. Part 3 covers genome organization, and here there is a sad reflection of the state of work on eukaryotic DNA replication which takes up only 13 pages, while in contrast the next chapter on organelle genomes occupies 55 pages. The final part is an account of the expression of genetic information and this section provides frequent illustrations of the book's principal strength, namely that it is not just a catalogue, but that it provides a level of critical discussion expected of a specialist review. Sustaining this penetrating standard of analysis for more than 900 pages of text is a remarkable achievement. The cautious judgements of controversial issues will ensure that the book remains valuable for many years, even though the subject matter continues to evolve rapidly. Unfortunately, however, the continued caution and criticism sometimes distract from the main thrust of the book.

The problem of extracting principal messages and key concepts from such a detailed discussion could be overcome by providing summary or conclusion sections. The final few pages of the book go some way towards this objective, but I feel that a work of this size would benefit greatly from a summary of the key points from each chapter. Because it is difficult to obtain clear overviews of the subject from the book, I would not recommend it for a novice student whose interest in the field has not been aroused already; its scope and scale could be simply overwhelming. I believe the most serious fault of the book is this lack of contrast. Apart from the addition of summaries, a more frequent use of diagrams to illustrate key concepts might also improve the emphasis. That said, the diagrams which are provided are effective, as are the tables which condense large amounts of information into a readily digestible form. The few photographs are also well chosen to provide appropriate illustration of structures.

Perhaps the most striking demonstration of the scope of this remarkable book is the 169 pages of references. Almost 4,000 papers are cited, making this an unusually valuable bibliographical source. Despite the large number of references cited they cause surprisingly little disruption to the text which remains fluent. Even more extraordinary is the fact that this is only one of the three volumes which comprise Gene Expression by this author; the other two deal with bacterial genomes (Vol.1) and plasmids and phages (Vol.3).

A potential flaw in a book which attempts such detailed coverage of such a wide field is factual error. However the author has maintained a high level of accuracy. The only faults of this nature I could find while reading the entire book were minor errors of detail rather than those of major facts or principles.

Although I would not recommend this book as an introduction to the subject, 1 feel that it is simply the best specialized discussion of the field that I have seen and I would recommend it enthusiastically to advanced students, teachers and researchers.

R.A. Laskey is a member of the Scientific Staff of the MRC Laboratory of Molecular Biology, Cambridge.

\section{Theories of conduction in organic solids}

\section{Martin R. Willis}

Electrical Transport in Solids. By Kwan C. Kao and Wei Hwang. Pp.663. ISBN 0-08-023973-0. (Pergamon: 1981.) £50, $\$ 120$.

ORGANIC solids exhibit the full range of electrical behaviour; they may be excellent insulators or superconductors. The polycyclic aromatic hydrocarbons, of which anthracene is typical, have been the most fully characterized and extensively studied, and are possibly the most fully understood. These materials are high-gap semiconductors or insulators, and band theory has been widely used for the interpretation of results. But since its applicability to materials with such narrow bands and low mobilities is questionable, other approaches such as hopping, tunnelling and polaron conduction have been applied.

The authors of this book have performed a valuable service by bringing together in a coherent form many of the theories applicable to conduction in organic solids, particularly polycyclic hydrocarbons. Much of the theory, particularly band theory, trapping, carrier injection and space charge limited currents, was originally derived for inorganic systems, but the limitations in its application to organic solids are stressed and numerous experimental results are quoted for such materials. Other theories, such as aspects of the exciton theory of photo-carrier generation were developed specially for molecular crystals. For those interested in solar energy conversion there is a section on photo-voltaic effects. The coverage is even greater than appears from the index, for Seebeck and Peltier effects are not listed but are well covered in the text.

A particularly helpful feature of the book is the presentation of such diverse theories using a common set of symbols, clearly defined at the beginning of the text, and mainly in SI units. Experimental results are, however, quoted as originally published and hence are mainly in cgs units. Energies are in electron volts, in line with current practice, but it is confusing that activation energy for conduction is defined in such a way as to make it equal to the band gap of an intrinsic semiconductor, in preference to the usual definition based on the Arrhenius equation.

Taken overall, this is an extremely welcome addition to the literature of the organic solid state and there will be few workers in the field who will not want a copy on their shelves, or at least in the library of their institution.

Martin R. Willis is Reader in Physical Chemistry at the University of Nottingham. 